\title{
Local Treatment with Triamcinolone Acetonide and Bevacizumab for Ocular Symptoms in a Patient with POEMS Syndrome
}

\author{
Marcin G. Prost ${ }^{a} \quad$ H. Jacobus Gilhuis ${ }^{b} \quad$ Rolf E. Brouwer ${ }^{c}$ Piotr Gawda ${ }^{d}$ \\ Departments of ${ }^{\mathrm{a}}$ Ophthalmology, ${ }^{\mathrm{b}} \mathrm{Neurology}$ and ${ }^{\mathrm{c}} \mathrm{Haematology}$ and Oncology, Reinier \\ de Graaf Group, Delft, The Netherlands; ${ }^{d}$ Department of Rehabilitation and Physiotherapy, \\ Medical University of Lublin, Lublin, Poland
}

\section{Key Words}

POEMS syndrome $\cdot$ Optic disc oedema $\cdot$ Macular oedema $\cdot$ Bevacizumab $\cdot$ Triamcinolone

\begin{abstract}
Purpose: To report our experience in managing a case of bilateral optic disc oedema and unilateral cystoid macular oedema with a posterior subtenon (PST) injection of triamcinolone acetonide and intravitreal bevacizumab (IVB) in a patient with polyneuropathy, organomegaly, endocrinopathy, monoclonal gammopathy, and skin changes (POEMS) syndrome. Case Report: A 50-year-old healthy woman complaining of bilateral blurred vision for several days was referred to the ophthalmologist. An eye examination showed a bilateral optic disc swelling and small preretinal haemorrhages. In addition, a peripheral neuropathy was found. Laboratory tests showed elevated levels of M-protein type IgA of $1.4 \mathrm{~g} / \mathrm{l}$ and a vascular endothelial growth factor (VEGF) concentration of $6.1 \mathrm{ng} / \mathrm{ml}$. The cerebrospinal fluid protein content was $131 \mathrm{mg} / \mathrm{dl}$. An abdominal CT scan revealed hepatosplenomegaly. The diagnosis of POEMS syndrome was made based on the polyneuropathy, hepatosplenomegaly, IgA lambda monoclonal proteinemia, and optic disc swelling. As vision declined, $40 \mathrm{mg}$ PST injection of triamcinolone was given bilaterally. Ten weeks thereafter, 2 IVB injections of 2.5 $\mathrm{mg}$ were administered to the left eye, with a 4-week interval. Because of an incomplete recovery of her visual functions, diarrhoea and general malaise, melphalan, followed by autologous peripheral blood stem cell transplantation, was initiated. After systemic treatment, she had no visual complaints anymore, the optic disc oedema had resolved, and the VEGF serum concentration had normalized. Discussion: Local treatment with IVB and PST injection of triamcinolone can be an option for ocular symptoms in POEMS syndrome.
\end{abstract}

Marcin G. Prost MD

Department of Ophthalmology

Reinier de Graaf Group, Reinier de Graafweg 3-11

NL-2625 AD Delft (The Netherlands)

E-Mailm.prost@rdgg.nl 
Prost et al.: Local Treatment with Triamcinolone Acetonide and Bevacizumab for Ocular Symptoms in a Patient with POEMS Syndrome

However, given the incomplete recovery of the ocular abnormalities and the other symptoms, systemic treatment remains standard.

(c) 2014 S. Karger AG, Basel

\section{Case Report}

A 50-year-old woman was referred to us for bilateral blurred vision as well as dryness and irritation of her eyes for several days. Except for a mild fatigue and a slight headache, she had no further complaints. Her visual acuity was 1.0 in both eyes, and slit-lamp examination was normal. A fundus examination revealed bilateral optic disc swelling and small preretinal peripapillary splinter haemorrhages in the right eye (fig. 1a). Her colour vision was normal (13/13 on Ishihara). Humphrey visual field testing showed an enlarged blind spot and an inferonasal deficit in both eyes (right > left) (fig. 1). Fluorescein angiography demonstrated a bilateral late leakage of dye from the optic disc and pooling consistent with cystoid macular oedema (CMO) in the left eye. Non-perfused areas, intraretinal microvascular abnormalities and retinal neovascularizations were not seen. Optical coherence tomography (OCT) performed with Cirrus HD-OCT 6.0 showed an increased macular thickness with a small amount of subretinal fluid at the fovea in the left eye. In the right eye, the macular thickness was normal (fig. 2). The segmentation algorithm was not used. The B-scan ultrasonography demonstrated disc swelling, but no drusen (fig. 1).

General physical examination was normal except for absent ankle reflexes and a diminished sensation in the feet. Laboratory tests showed an erythrocyte sedimentation rate of 30 $\mathrm{mm} / \mathrm{h}$, elevated levels of M-protein type IgA of $1.4 \mathrm{~g} / \mathrm{l}$, and a vascular endothelial growth factor (VEGF) concentration of $6.1 \mathrm{ng} / \mathrm{ml}(\mathrm{n}=0.028-0.966 \mathrm{mg} / \mathrm{l})$. Cerebrospinal fluid pressure was normal, and the cerebral spinal fluid had a protein content of $131 \mathrm{mg} / \mathrm{dl}$ (normal $<45 \mathrm{mg} / \mathrm{dl}$ ). An abdominal CT revealed hepatosplenomegaly. Her EMG showed a mild axonal sensorimotor polyneuropathy. The diagnosis of polyneuropathy, organomegaly, endocrinopathy, monoclonal gammopathy, and skin changes (POEMS) syndrome was made based on polyneuropathy, hepatosplenomegaly, IgA lambda monoclonal proteinemia, and the optic disc swelling. Additional investigations to find a further possible malignancy revealed no abnormalities.

After 2 months, local treatment with $40 \mathrm{mg}$ posterior sub-Tenon (PST) injection of triamcinolone in both eyes was started because of continuous complaints of blurred vision. This was followed by 2 intravitreal bevacizumab (IVB) injections of $2.5 \mathrm{mg}$ in the left eye at intervals of 4 weeks after 10 weeks. Local therapy with PST injection had resulted in a small decrease of the optic disc swelling after 4 weeks. In the left eye, the macular oedema regressed, and the central retinal thickness, as measured by OCT, diminished. The IVB treatment resulted in a slight regression of the optic disc oedema (ODO) and a minimal decrease of the CMO (table 1). Because of diarrhoea and general malaise and the fact that the blurred vision had diminished but not completely disappeared, melphalan was initiated. This was followed by autologous peripheral blood stem cell transplantation. After systemic treatment, the patient had no further visual complaints and the ODO had disappeared (fig. 3). The VEGF serum concentration normalized. Three years after the initial diagnosis, the patient's ophthalmological condition remained unchanged. 
Prost et al.: Local Treatment with Triamcinolone Acetonide and Bevacizumab for Ocular Symptoms in a Patient with POEMS Syndrome

\section{Discussion}

POEMS syndrome is a rare and chronic paraneoplastic disorder characterized by polyneuropathy, organomegaly, endocrinopathy, M-protein and skin changes, resulting from an underlying plasma cell disorder. The acronym refers to some, but not all the features. POEMS syndrome is potentially fatal with a substantial deterioration in quality of life and a chronic clinical course $[1,2]$. Eye involvement is characterized by ODO and CMO. Vision acuity is mostly normal. ODO, unilateral or bilateral, is present in $29-55 \%$ of patients [2, 3]. Macular oedema secondary to optic disc leakage is rare and associated with macular detachment $[4$, 5]. These patients usually report blurred vision [2-6]. In a study of 33 POEMS patients undergoing ophthalmologic examination, 5 patients reported diplopia, 15 patients had blurred vision, and 3 patients had ocular pain. Bilateral ODE was seen in about half of the cases, with about one third asymptomatic at the first examination [6].

The pathogenesis of POEMS syndrome is not fully understood. An elevated production of proangiogenic and proinflammatory cytokines are believed to play a central role. VEGF and other cytokines are elevated, reflecting the clinical condition $[1,7]$.

The mechanism underlying the formation of ODO and CMO associated with POEMS syndrome is unclear. ODO can result either from increased intracranial pressure or from a chronic overproduction of cytokines (such as VEGF) which supports extravascular volume overload secondary to raised vascular permeability [7-9]. Development of CMO might be attributed to the elevated serum VEGF and not to the VEGF secreted from retinal tissues [10]. ODO and CMO may result from an osmotic gradient created by the deposition of highmolecular-weight immunoglobulins within and beneath the neurosensory retina [7].

Anti-VEGF antibodies such as bevacizumab are an effective treatment for ocular conditions associated with increased VEGF levels. Theoretically, they could also be used locally [1, 8]. Our case is the first in which bevacizumab was used intravitreally, resulting (in combination with PST injection) in a partial regression of ODO and CMO. ODO and CMO normalized however only after systemic treatment. Because POEMS is a systemic condition, the ocular inhibition of VEGF alone is insufficient. Additionally, decreasing VEGF serum concentration as a response to systemic treatment correlated with the resolving of optic disc swelling.

PST has been widely used in ophthalmology for the treatment of intraocular proliferative, oedematous, and neovascular diseases. A reduction of macular oedema can be induced by the anti-inflammatory reaction of steroids as investigated in several studies $[9,10]$. In a case report describing pars plana vitrectomy and intraocular triamcinolone injections as a treatment for $\mathrm{CMO}$, the macular thickness decreased. In the untreated eye, the macular thickness altered in relation to the serum VEGF level [11].

In conclusion, local treatment with IVB and PST injection of triamcinolone can be an option for ocular symptoms in POEMS syndrome, but always in combination with the standard treatment of this syndrome.

\section{Disclosure Statement}

The authors declare no conflict of interest. 
Prost et al.: Local Treatment with Triamcinolone Acetonide and Bevacizumab for Ocular Symptoms in a Patient with POEMS Syndrome

\section{References}

1 Straume O, Bergheim J, Ernst P: Bevacizumab therapy for POEMS syndrome. Blood 2006;107:4972-4974.

-2 Nakanishi T, Sobue I, Toyokura Y, Nishitani H, Kuroiwa I, Satoyoshi E, Tsubaki T, Igata A, Ozaki Y: The CrowFukase syndrome: a study of 102 cases in Japan. Neurology 1984;34:712-720.

-3 Chong DY, Comer GM, Trobe JD: Optic disc edema, cystoid macular edema, and elevated vascular end othelial growth factor in a patient with POEMS syndrome. J Neuroophthalmol 2007;27:180-183.

-4 Gutierrez-Ortiz C, Castro-Rebollo M, Pareja J, Beckford C, Teus M: Bilateral non-simultaneous optic neuropathy and unilateral macular edema in a patient with POEMS syndrome. Eur J Ophthalmol 2009;19:166-169.

5 Okada K, Yamamoto S, Tsuyama Y, Mizunoya S: Case of POEMS syndrome associated with bilateral macular detachment resolved by autologous peripheral blood stem cell transplantation. Jpn J Ophthalmol 2007;51:237-238.

-6 Kaushik M, Pulido JS, Abreu R, Amselem L, Dispenzieri A: Ocular findings in patients with polyneuropathy, organomegaly, endocrinopathy, monoclonal gammopathy, and skin changes syndrome. Ophthalmology 2011;118:778-782.

7 Watanabe 0, Arimura K, Kitajima I, Osame M, Maruyama I: Greatly raised vascular endothelial growth factor (VEGF) in POEMS syndrome. Lancet 1996;347:702.

$>8$ Badros A, Porter N, Zimrin A: Bevacizumab therapy for POEMS syndrome. Blood 2005;106:1135.

-9 Qi HP, Bi S, Wei SQ, Cui H, Zhao JB: Intravitreal versus subtenon triamcinolone acetonide injection for diabetic macular edema: a systematic review and meta-analysis. Curr Eye Res 2012;37:1136-1147.

-10 Shen L, You Y, Sun S, Chen Y, Qu J, Cheng L: Intraocular and systemic pharmacokinetics of triamcinolone acetonide after a single 40-mg posterior subtenon application. Ophthalmology 2010;117:2365-2371.

11 Imai H, Kusuhara S, Nakanishi Y, Teraoka Escaño MF, Yamamoto H, Tsukahara Y, Negi A: A case of POEMS syndrome with cystoid macular edema. Am J Ophthalmol 2005;139:563-566.

Table 1. Central retinal thickness and cube average thickness measured with OCT

\begin{tabular}{|c|c|c|c|c|c|c|}
\hline & \multicolumn{3}{|l|}{ Right eye } & \multicolumn{3}{|l|}{ Left eye } \\
\hline & $\begin{array}{l}\text { central subfield } \\
\text { thickness, } \mu \mathrm{m}\end{array}$ & $\begin{array}{l}\text { cube } \\
\mathrm{mm}^{3}\end{array}$ & $\begin{array}{l}\text { e,cube average } \\
\text { thickness, } \mu \mathrm{m}\end{array}$ & $\begin{array}{l}\text { central subfield } \\
\text { thickness, } \mu \mathrm{m}\end{array}$ & $\begin{array}{l}\text { cube } \\
\mathrm{mm}^{3}\end{array}$ & $\begin{array}{l}\text {, cube average } \\
\text { thickness, } \mu \mathrm{m}\end{array}$ \\
\hline Before treatment & 324 & 12.0 & 334 & 341 & 12.5 & 348 \\
\hline After PST injection & 296 & 9.5 & 263 & 299 & 9.8 & 273 \\
\hline After IVB & - & - & - & 291 & 10.5 & 292 \\
\hline After local and systemic treatment & 299 & 9.6 & 266 & 293 & 10.0 & 278 \\
\hline
\end{tabular}


Case Reports in

Ophthalmology

\begin{tabular}{l|l}
\hline Case Rep Ophthalmol 2014;5:416-422 \\
\hline DOI: $10.1159 / 000369856$ & $\begin{array}{l}\text { C 2014 S. Karger AG, Basel } \\
\text { www.karger.com/cop }\end{array}$ \\
\hline
\end{tabular}

Prost et al.: Local Treatment with Triamcinolone Acetonide and Bevacizumab for Ocular Symptoms in a Patient with POEMS Syndrome
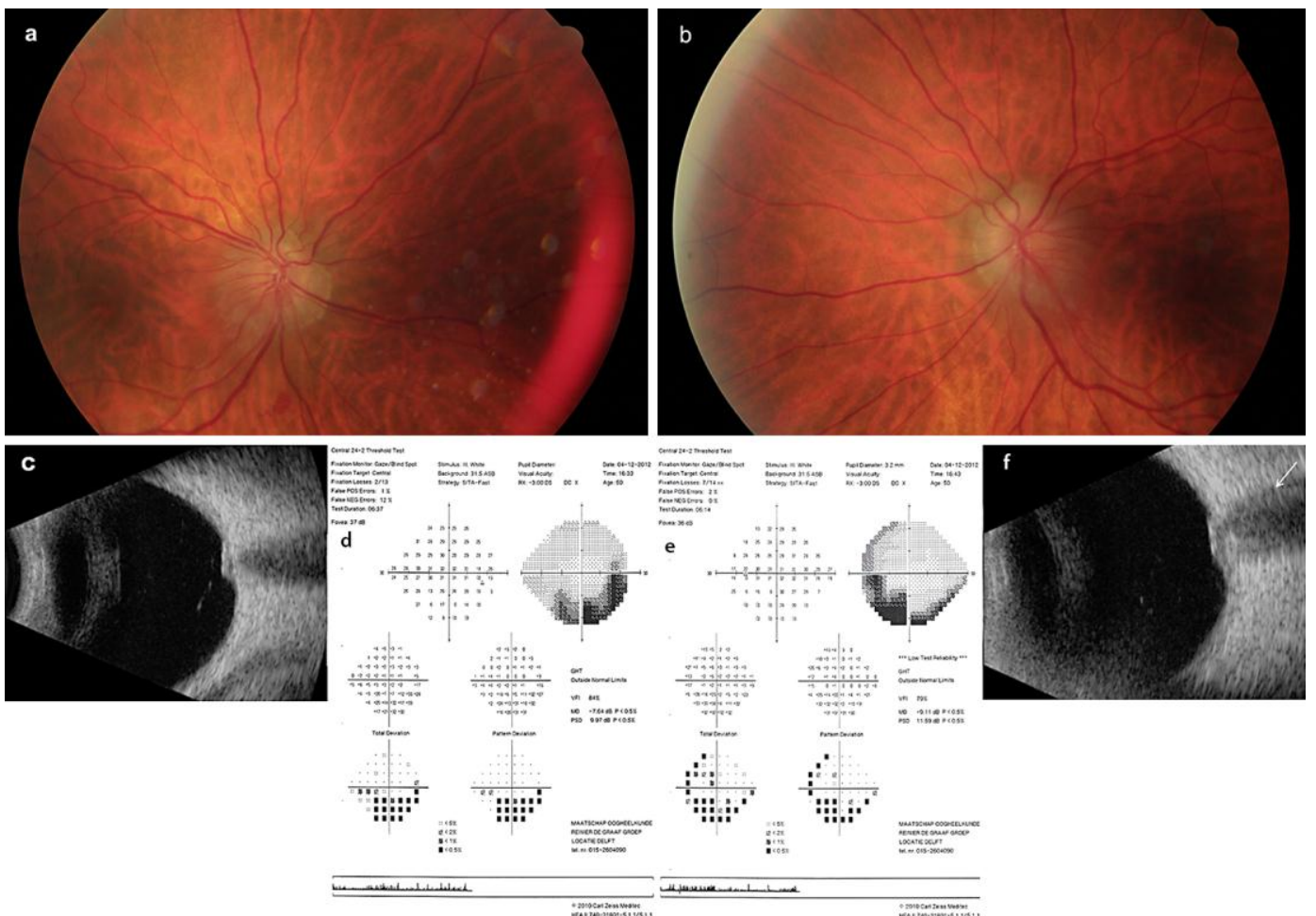

Fig. 1. A fundus photograph of the right (a) and the left eye (b) with bilateral optic disc swelling. An ophthalmologic echography of the right (c) and the left eye (f): sagittal section of the optic nerve with visible disc oedema and small enlargement of the subdural space in the left eye (white arrows). The visual field of the right (d) and the left eye (e) was performed with Humphrey visual field using a 30-2 algorithm with SITA fast strategy. It shows abnormally enlarged blind spots and inferonasal deficits in both eyes. 
Prost et al.: Local Treatment with Triamcinolone Acetonide and Bevacizumab for Ocular Symptoms in a Patient with POEMS Syndrome

\section{A. Before treatment}
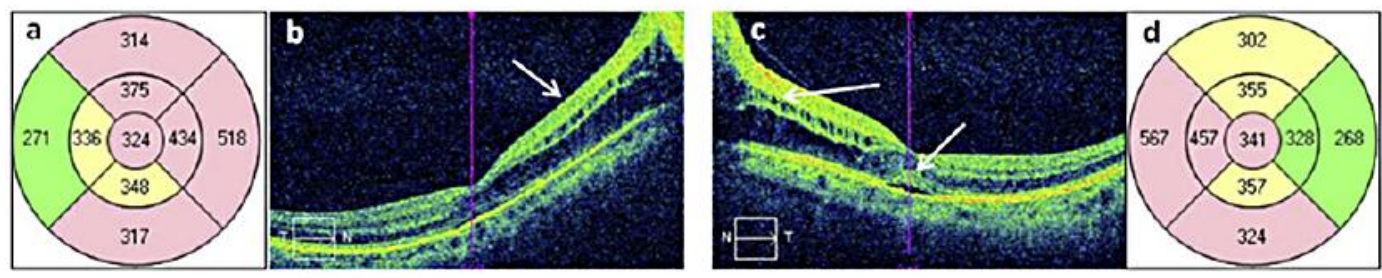

B. After the local PST treatment
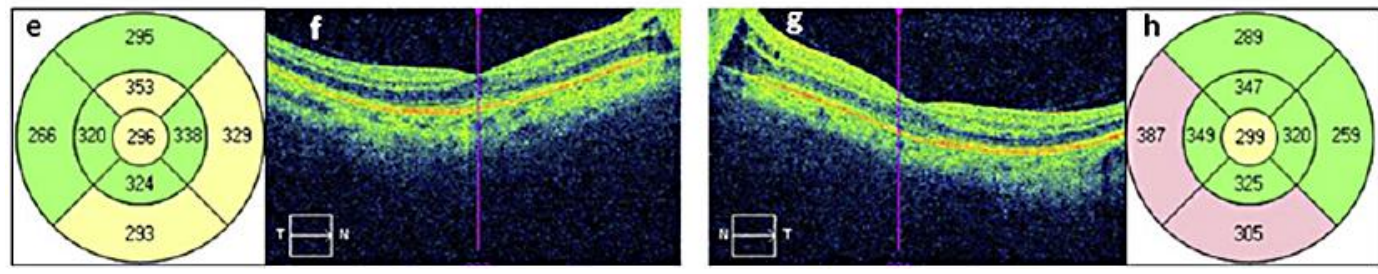

Fig. 2. A macular thickness map with OCT image of the macula of the right (a, b) and the left eye (c, d) before treatment. Illustrations $\mathbf{b}$ and $\mathbf{d}$ show a cystoid fluid accumulation in the outer plexiform layer in the peripapillary area, and a subretinal fluid accumulation under the fovea (white arrows). The macular thickness map with OCT image of the macula of the right $(\mathbf{e}, \mathbf{f})$ and the left eye $(\mathbf{g}, \mathbf{h})$ after PST treatment. Illustrations $\mathbf{f}$ and $\mathbf{h}$ show a decrease in cystoid fluid accumulation in the outer plexiform layer in the peripapillary area as well as the subretinal fluid accumulation under the fovea. 
Prost et al.: Local Treatment with Triamcinolone Acetonide and Bevacizumab for Ocular Symptoms in a Patient with POEMS Syndrome

\section{A. After the local IVB treatment}
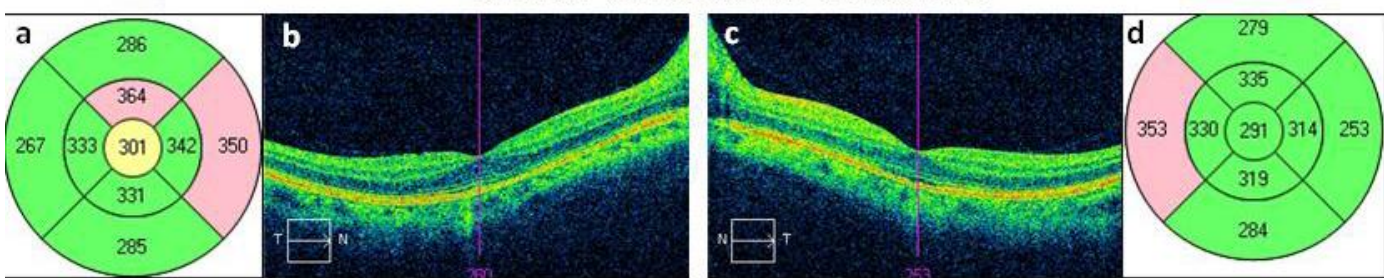

B. After the local and systemic treatment
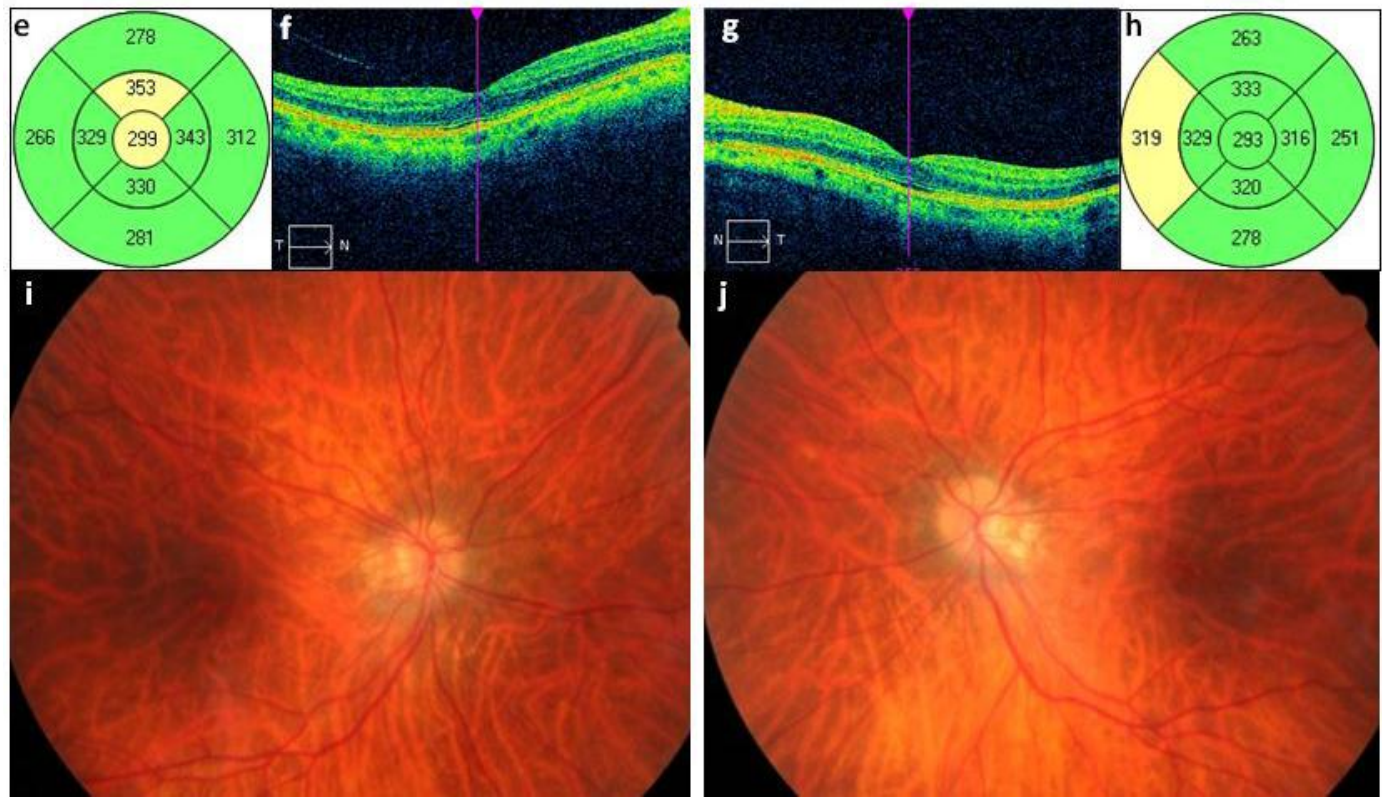

Fig. 3. A macular thickness map with OCT image of the macula of the right (a, b) and the left eye (c, d) after the local IVB treatment. Illustrations $\mathbf{b}$ and $\mathbf{d}$ show further resolving of the cystoid fluid accumulation in the retina and no macular oedema. The macular thickness map with OCT images of the macula of the right $(\mathbf{e}, \mathbf{f})$ and the left eye $(\mathbf{g}, \mathbf{h})$ after local and systemic treatment. Illustrations $\mathbf{f}$ and $\mathbf{h}$ show a stable central foveal thickness. A fundus photograph of the right (i) and the left eye (j) without optic disc swelling. 